\title{
Assessing the Quality of Visualization Metaphor of Fuzzy Cognitive Maps on the Basis of Formalized Cognitive Clarity Criteria
}

\author{
A.G. Podvesovskii ${ }^{1}$, R.A. Isaev ${ }^{1}$ \\ apodv@tu-bryansk.ru|ruslan-isaev-32@yandex.ru \\ ${ }^{1}$ Bryansk State Technical University, Bryansk, Russia
}

\begin{abstract}
The paper presents continuation of research in the field of constructing a visualization metaphor of cognitive models based on fuzzy cognitive maps. The focus is on the spatial metaphor as the basis for representation metaphor formation. A method is proposed for quality assessment of a spatial metaphor of a fuzzy cognitive map based on formalized cognitive clarity criteria defined in the previous part of the study. To this end, methods have been developed to formalize several nontrivial criteria of cognitive clarity. An example is given that confirms correctness of the proposed method for assessing the quality of a visualization metaphor.
\end{abstract}

Keywords: fuzzy cognitive map, graph visualization, cognitive clarity, visualization metaphor.

\section{Introduction}

This paper continues a series of publications of authors' research materials in the field of visualization of cognitive models based on fuzzy cognitive maps (FCM). A FCM reflects researcher's subjective idea of a system in the form of a set of semantic categories (called factors or concepts) and a set of causal relationships between them $[2,8]$. Thus, a FCM can be graphically represented in the form of a weighted directed graph, the vertices of which correspond to concepts, and edges - to cause-and-effect relationships.

One of the conditions for effective work with a cognitive model is to ensure its visual representation. In [4], the authors proposed an approach to FCM visualization based on reducing this problem to a graph visualization problem. Later in [7], this approach was expanded by using the concept of visualization metaphor and its two components - spatial metaphor and representation metaphor [10]. FCM visualization metaphor is based on graph visualization algorithms $[5,6]$ and cognitive clarity concept, which characterizes the ease of intuitive understanding of information [1] and takes into account the problem of human's limited cognitive abilities when reading graphs (a detailed analysis of this problem can be found, for example, in [3]). Thus, a link has been discovered between the quality of FCM visualization metaphor and the level of cognitive clarity of the resulting visual image: the higher the level of cognitive clarity provided by the visualization metaphor, the simpler is the process of expert understanding of the cognitive model in its visual analysis. To assess the level of cognitive clarity, a set of criteria is proposed. It is concluded that cognitive clarity criteria are the means of the most natural evaluation of visualization metaphor quality. The present work is devoted to the development of a method for such an assessment. The previous part of the study focused on the representation metaphor, whereas this work focuses on the spatial metaphor, which is the basic component of the visualization metaphor and serves as the foundation for the subsequent formation of the representation metaphor.

\section{Methods of formalizing cognitive clarity criteria}

As noted in [7], the result of applying the spatial metaphor of FCM visualization is the location of the cognitive graph (i.e., coordinates of its vertices and edges) on the plane, which is optimal in the sense of cognitive clarity criteria, that is, contributing to the quality increase of the resulting visual image. Note that the cognitive clarity criteria are formulated at a qualitative level using natural language. At the same time, the possibility of spatial metaphor automated quality assessment is of interest. In this connection, the verification of the resulting visual image for compliance with the criteria of cognitive clarity must be implemented algorithmically. To this end, formalized representation of the cognitive clarity criteria is required.

By formalizing a certain cognitive clarity criterion, we mean developing methods, techniques and algorithms that allow determining a numerical score for a visual image of an arbitrary cognitive map characterizing the extent to which this image complies with the selected criterion. Formalization of some criteria (minimizing the length of edges, minimizing the number of edge crossings, minimizing the number of curved edges) is trivial, and its description is of no interest. Let us consider possible ways of formalizing several nontrivial cognitive clarity criteria.

\subsection{Optimizing edge directions}

This criterion is based on the observation that laying out edges in the directions "from top to bottom" and "from left to right" helps to accelerate "reading" of a FCM in comparison with the orientation of edges in the opposite directions. We shall call the directions that facilitate faster "reading" of FCMs as well as edges having such directions convenient. As an example, we can compare two visual images of a cognitive graph in Figure 1.
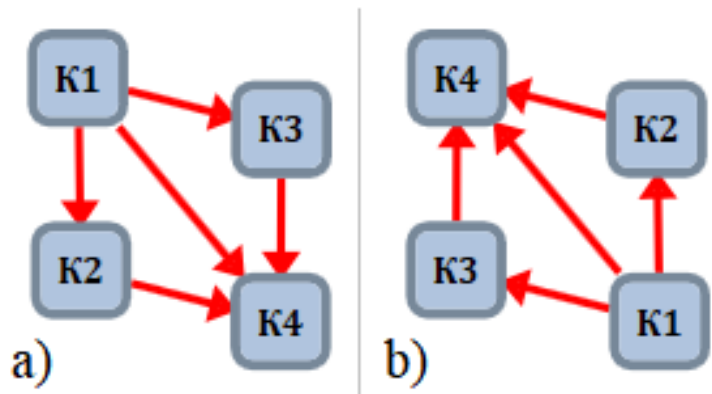

Fig. 1. Examples of visual images with convenient (a) and not convenient (b) directions of edges

Apparently, convenient directions coincide with the direction of reading, adopted in a particular language culture. Therefore, other conditions being equal, preference should be given to visual images containing a greater number of convenient edges. It should be borne in mind that the described property is inherently fuzzy. So, edge orientation "from top to bottom" and "from right to left" can be considered partially convenient, since one of the usual directions of reading is preserved. Therefore, the mathematical apparatus of the fuzzy set theory can be used to formalize the criterion in question.

Let $A$ be a fuzzy set formalizing the concept of a "convenient edge direction". In order to set its membership function, let us define the edge direction as angle $\alpha$ between the 
vector drawn from the beginning of the edge to its end and the positive direction of the horizontal axis OX. Then membership function $\mu_{A}(\alpha)$ must satisfy the following requirements:

1) $\mu_{A}(\alpha)=1$ when $-\frac{\pi}{2} \leq \alpha \leq 0$;

2) $\mu_{A}(\alpha)=0$ when $\frac{\pi}{2} \leq \alpha \leq \pi$;

3) $0<\mu_{A}(\alpha)<1$ when $0<\alpha<\frac{\pi}{2}$ и $-\pi<\alpha<-\frac{\pi}{2}$;

4) increases monotonically on the interval $-\pi<\alpha<-\frac{\pi}{2}$;

5) decreases monotonically on the interval $0<\alpha<\frac{\pi}{2}$.

Given these requirements, $\mu_{A}(\alpha)=\cos (\alpha)$ can be accepted on the interval $0<\alpha<\frac{\pi}{2}$, and $\mu_{A}(\alpha)=-\sin (\alpha)$ can be accepted on the interval $-\pi<\alpha<-\frac{\pi}{2}$.

Having determined for each FCM edge the degree of its membership to set $A$, we can obtain a value characterizing the overall score of the entire visual image by this criterion - for example, as the average value of membership degrees of all edges.

Influence intensities should also be taken into account in the final assessment, since providing convenient directions for more significant influences is more important than for less significant ones. Therefore, absolute values of influence intensities can be used as weighting coefficients and membership values of the corresponding edges can be multiplied by them when calculating the average value.

\subsection{Maximizing unidirectionality of consecutive edges}

This criterion is based on the idea that "reading" a FCM will be faster if gaze direction has to be changed as little as possible during the process of viewing paths and cycles of a graph.

We will call two edges consecutive if one of them enters the vertex from which the other one starts. Thus, any path and cycle of a graph consists of pairs of consecutive edges. Therefore, in accordance with this criterion, preference should be given to visual images with a greater number of pairs of consecutive edges depicted unidirectionally. For example, let us compare two visual images of a fragment of some FCM (Fig. 2).

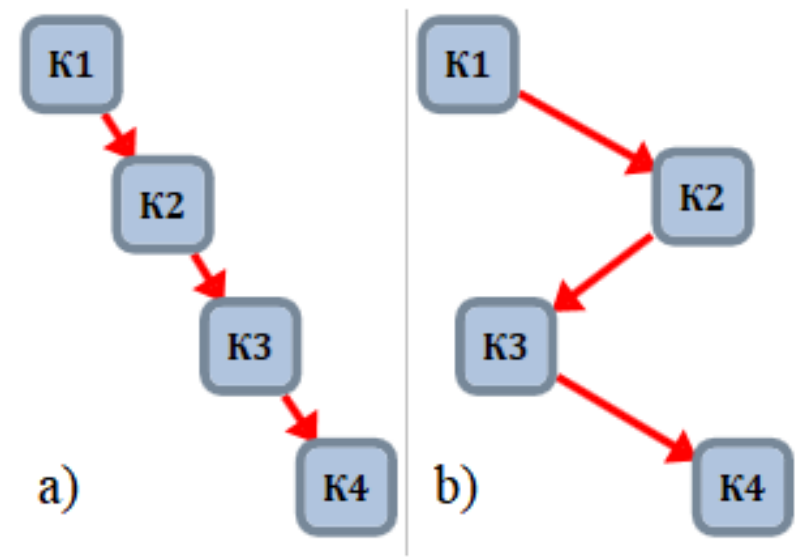

Fig. 2. Examples of visual images with unidirectional (a) and bidirectional (b) consecutive edges
Obviously, the unidirectional property is fuzzy. Suppose $B$ is a fuzzy set formalizing the concept of unidirectional edges. The membership degree of a pair of consecutive edges to set $B$ is determined by angle $\beta \in[0, \pi]$ between these edges. We assume that changing gaze direction by 90 degrees or more slows down the process of viewing the path in the graph significantly. Accordingly, the following requirements are imposed on the membership function $\mu_{B}(\beta)$ :

1) $\mu_{B}(\beta)=1$ when $\beta=0$;

2) $\mu_{B}(\beta)=0$ when $\beta \geq \frac{\pi}{2}$;

3) $0<\mu_{B}(\beta)<1$ when $0<\beta<\frac{\pi}{2}$;

4) decreases monotonically on the interval $0<\beta<\frac{\pi}{2}$.

Given these requirements, on the interval $0<\beta<\frac{\pi}{2}$ we accept $\mu_{B}(\beta)=\cos (\beta)$.

By analogy with the previous criterion, the score of the entire visual image by the criterion under study can be found as the average value of membership degrees of all pairs of consecutive edges to set $B$. Influence intensities can also be taken into account in a similar way.

\subsection{Maximizing graph symmetry}

Due to the fact that FCM structure reflects the structure of a simulated system it is important to ensure the symmetry of FCM visual image to increase its cognitive clarity. Thus, symmetries of a graph image help to detect symmetries inherent in the system itself.

Let us consider various aspects of determining degree of image symmetry in relation to FCM visual image.

Firstly, the following types of symmetries are the simplest to perceive and, therefore, of greatest practical interest:

1) axial symmetry with respect to the horizontal axis of an image;

2) axial symmetry with respect to the vertical axis of an image;

3) central symmetry with respect to the geometric center of an image.

Secondly, in the case of an FCM, as well as any digraph, the following levels of symmetry can be distinguished (Fig. 3 considers the case of symmetry about the vertical axis):

1) lack of symmetry at the level of any elements of the graph (Fig. 3, a);

2) at the level of vertices excluding edges (Fig. 3, b);

3) at the level of edges excluding their directions (Fig. 3, c);

4) at the level of edges including their directions (Fig. 3, d).

The above example allows for the conclusion that symmetry at the vertex level does not bring any tangible effect to increasing cognitive clarity of an FCM visual image. Thus, only symmetry at the level of edges is of practical interest.

Thirdly, it is obvious that in addition to strict symmetry (Fig. 4, a), we can also speak of approximate symmetry (Fig. 4, b), which can be represented as a certain deviation from the strict one.

With this in mind, the degree of symmetry of an FCM visual image can be defined as a measure of its proximity to a strictly symmetric image. Thus, it is necessary to develop an algorithm that is able to determine the degree of symmetry for an arbitrary image taking into account a given type and level of symmetry. 


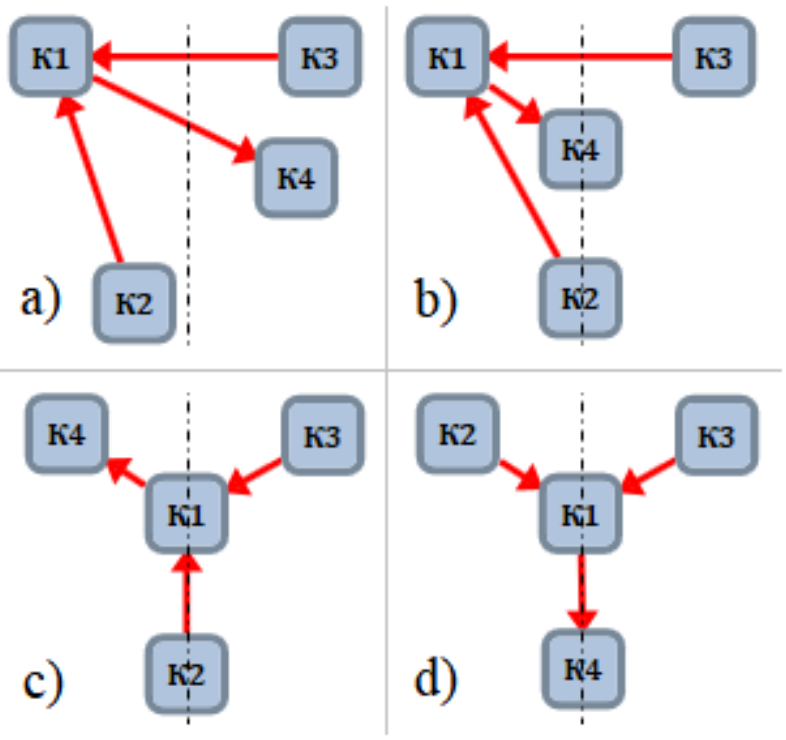

Fig. 3. Examples of different symmetry levels of FCM visual image (about the vertical axis)

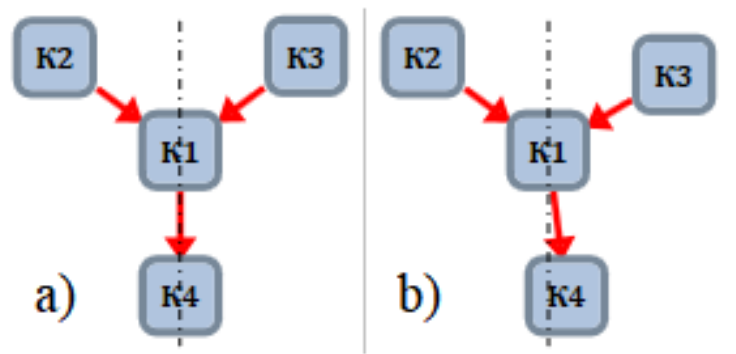

Fig. 4. Examples of strict (a) and approximate (b) symmetry of FCM visual image

The main idea of the proposed algorithm is as follows. For each element of an FCM visual image, the position of its "reflection" relative to a given axis or center is calculated. Next, for each of the "reflections", the element closest to it (in the sense of the chosen metric, for example, Euclidean distance) is selected from among all the elements of the image. Distances (in the selected metric) from all "reflections" to their nearest elements are added up. The resulting value characterizes the degree of symmetry of the visual image in question and has the following properties:

1) it is equal to 0 if the image has strict symmetry of a given (or stronger) level and type;

2) it is greater than 0 in all other cases;

3) it increases as the image becomes less and less symmetrical;

4) it does not have an upper bound since there is no "maximally asymmetric" image.

\section{Defining spatial metaphor quality assessment}

In [7], it was noted that many of cognitive clarity criteria contradict each other and it is impossible in the general case to ensure that FCM visual image meets all the criteria at the same time from an algorithmic point of view. Thus, it is necessary to develop decision rules modelling various forms of compromise among the criteria.

Of primary interest is the class of rules based on various types of criteria aggregations, primarily, sum and product ones. At the same time, there is reason to believe that the structure of relationships among cognitive clarity criteria is quite complex and is characterized by the following features:

1) criteria may exist that determine quality of a metaphor not separately but in combination with some other criteria;
2) in the whole set of criteria, there may be several "bundles of criteria" affecting metaphor quality independently of each other.

To formalize the described assumption, we shall accept that set of criteria $K=\left\{k_{1}, \ldots, k_{n}\right\}$ can be divided into disjoint subsets $G_{1}, \ldots, G_{m}$. Further, we will also assume that FCM visual image scores by all criteria take their values in the interval $[0,1]$.

For each criteria subset $G_{i}$, we shall introduce value $g_{i} \in[0,1]$ - visual image score for this subset. We shall specify the following requirements for such a score:

1) if the image score by at least one criterion from subset $G_{i}$ is 0 , then $g_{i}=0$;

2) $g_{i}=1$ if and only if the image score by all criteria from subset $G_{i}$ is 1 ;

3) if the image score according to all criteria from subset $G_{i}$ is $a$, then $g_{i}=a$ (idempotency).

One of the operations meeting the specified requirements is a weighted product aggregation:

$$
g_{i}=\prod_{j=1}^{l} m_{j}^{w_{j}},
$$

where $m_{j}$ is image score by the $j$-th criterion from $G_{i}$, $w_{j} \in[0,1]$ is relative importance of the $j$-th criterion within $G_{i}$ $\left(w_{1}+\ldots+w_{l}=1\right), l$ is power of $G_{i}$.

To obtain final score, we shall apply weighted sum aggregation to the scores obtained for all subsets:

$$
F=\sum_{i=1}^{m} w_{g_{i}} g_{i},
$$

where $w_{g_{i}} \in[0,1]-$ is relative importance of subset $G_{i}$ $\left(w_{g_{1}}+\ldots+w_{g_{m}}=1\right)$.

Moreover, value $w_{g_{i}}$ can be interpreted as FCM visual image score that fully satisfies the subset of criteria $G_{i}$ and does not completely satisfy other subsets of criteria.

Thus, for the final score $F$, the following properties are guaranteed:

1) $F \in[0,1]$;

2) $F=1$ if scores for all criteria are 1 ;

3) $F=0$ if scores for all subsets of criteria $G_{1}, \ldots, G_{m}$ are 0 (i.e., at least one criterion scored with 0 is present in each subset).

\section{An example of spatial metaphor quality assessment}

Let us conduct experimental verification of the proposed method for quality assessment of FCM spatial metaphor. In view of this, we shall consider three different visual images of a certain FCM presented in Fig. 5-7.

Assessment of the degree of visual images compliance with cognitive clarity criteria has been performed using the proposed methods of formalizing these criteria. Table 1 shows criteria scores of images normalized to the range $[0,1]$. When normalizing the scores, the initial need to minimize a number of criteria was taken into account. Thus, after normalization, all criteria must be maximized. 


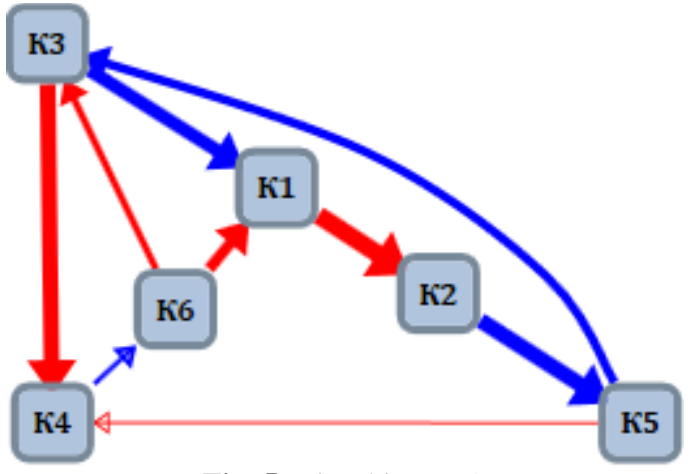

Fig. 5. Visual image 1

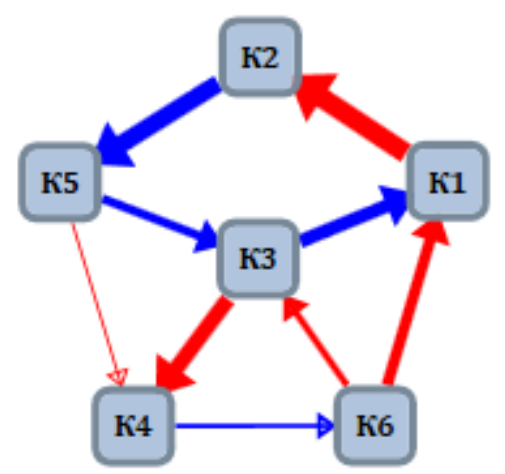

Fig. 6. Visual image 2

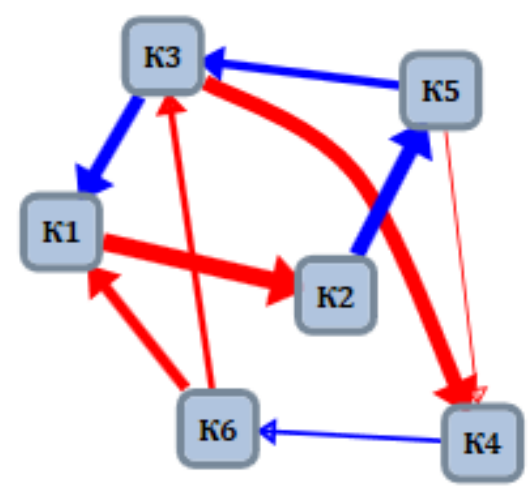

Fig. 7. Visual image 3

Table. 1. Visual image criteria scores

\begin{tabular}{|l|c|c|c|}
\hline \multirow{2}{*}{\multicolumn{1}{|c|}{ Criterion }} & \multicolumn{3}{c|}{ Visual image number } \\
\cline { 2 - 4 } & $\mathbf{1}$ & $\mathbf{2}$ & $\mathbf{3}$ \\
\hline 1. Optimizing placement area & 0 & 1 & 0.88 \\
\hline 2. Minimizing edge lengths & 0 & 1 & 0.25 \\
\hline 3. Unifying edge lengths & 0 & 1 & 0.58 \\
\hline 4. Optimizing edge directions & 1 & 0 & 0.06 \\
\hline 5. Minimizing edge crossing & 1 & 1 & 0 \\
\hline $\begin{array}{l}\text { 6. Minimizing the number of } \\
\text { curved edges }\end{array}$ & 0.43 & 1 & 0 \\
\hline $\begin{array}{l}\text { 7. Maximizing } \\
\text { unidirectionality of } \\
\text { consecutive edges }\end{array}$ & 1 & 0.39 & 0 \\
\hline $\begin{array}{l}\text { 8. Maximizing graph } \\
\text { symmetry }\end{array}$ & 0 & 1 & 0.25 \\
\hline $\begin{array}{l}\text { 9. Maximizing angles } \\
\text { between incident edges }\end{array}$ & 0.16 & 1 & 0 \\
\hline
\end{tabular}

Let us demonstrate the use of various decision rules to obtain final scores for cognitive clarity levels of FCM visual images.

First, we shall apply a simple sum aggregation of criteria. Suppose the criteria priorities are set as follows: $w_{1}=0.2$; $w_{2}=w_{3}=w_{6}=w_{9}=0,05 ; w_{4}=w_{7}=w_{8}=0.1 ; w_{5}=0.3$. In this case, we obtain the following scores of cognitive clarity levels of visual images: $F_{1}=0.53 ; F_{2}=0.84 ; F_{3}=0.25$. Accordingly, the metaphor forming image 2 should be recognized most qualitative.

Now we shall adjust the criteria priorities increasing relative significance of the criteria of optimizing edge direction and maximizing unidirectionality of consecutive edges: $w_{1}=w_{2}=w_{3}=w_{6}=w_{8}=w_{9}=0,05 ; \quad w_{4}=w_{7}=0.2 ; \quad w_{5}=0.3$. Scores of cognitive clarity levels will change and take values $F_{1}=0.73 ; F_{2}=0.68 ; F_{3}=0.11$. In this case, the metaphor forming image 1 , which meets the highest priority criteria more than image 2, proves to be most qualitative.

Now, let us apply the decision rule proposed in this paper. Suppose that, based on some expert considerations, the set of criteria has been divided into two subsets: $G_{1}=\left\{k_{1}, k_{2}, k_{3}, k_{8}\right\}$ and $G_{2}=\left\{k_{4}, k_{5}, k_{6}, k_{7}, k_{9}\right\}$, whose priorities were initially set to 0.6 and 0.4 respectively. For simplicity, we will assume that the criteria priorities within the corresponding subsets are distributed evenly, that is 0.25 and 0.2 for the criteria from $G_{1}$ and $G_{2}$, respectively. We will obtain the following scores of cognitive clarity levels of visual images: $F_{1}=0.23 ; F_{2}=0.6$; $F_{3}=0.25$. The metaphor forming image 2 , which fully satisfies all the criteria from the higher priority subset of $G_{1}$, is recognized as the most qualitative.

Now let us set priorities $G_{1}$ and $G_{2}$ equal to 0.3 and 0.7 , respectively. In this case, scores of cognitive clarity levels will take values $F_{1}=0.41 ; \quad F_{2}=0.3 ; \quad F_{3}=0.13$. The metaphor forming image 1 is now considered the most high-quality, since this image better than others satisfies a higher priority criteria subset $G_{2}$.

Thus, the use of the proposed decision rule allows for setting relative importance of a certain set of criteria at once. At the same time, by regulating criteria priorities within the framework of corresponding subsets, it is possible to provide a finer consideration of their influence on the score of these subsets.

\section{Conclusion}

The paper presents the development of an approach to constructing a visualization metaphor of fuzzy cognitive maps. A method for assessing the quality of FCM spatial metaphor based on formalized cognitive clarity criteria is proposed and methods for formalizing several such criteria, which are nontrivial, are described. An example is given confirming the correctness of the proposed metaphor quality assessment method: visual images with a higher level of cognitive clarity get higher scores. In addition, application of two different decision rules has been demonstrated, allowing for quality evaluation of a metaphor based on its scores by groups of cognitive clarity criteria as well as set priorities of these groups.

Let us indicate directions for further research.

The first one is developing methodology for automatic selection of the optimal spatial metaphor of a FCM taking into account set priorities according to the cognitive clarity criteria; also implementation of the corresponding opportunity in IGLA decision support system, developed with the participation of the authors [9].

The second one is development of new decision rules for quality assessment of FCM metaphor allowing for more flexible consideration of user's specific preferences and acceptable forms of compromise among cognitive clarity criteria.

\section{Acknowledgments}

The reported study was funded by RFBR, project number 19-07-00844. 


\section{References}

[1] Abramova N.A., Voronina T.A., Portsev R.Y. O metodah podderzhki postroeniya i verifikacii kognitivnyh kart $\mathrm{s}$ primeneniem idej kognitivnoj grafiki [Ideas of Cognitive Graphics to Support Verification of Cognitive Maps] // Upravlenie bol'shimi sistemami. Special issue 30.1 "Setevye modeli v upravlenii". - Moscow: ICS RAS, 2010. - pp. 411-430 [in Russian].

[2] Borisov V.V., Kruglov V.V., Fedulov A.S. Nechetkie modeli i seti [Fuzzy Models and Networks]. - M.: Goryachaya Liniya - Telekom, 2012. - 284 p. [in Russian]

[3] Huang W., Hong S.H., Eades P. Predicting Graph Reading Performance: A Cognitive Approach // Proc. Asia Pacific Symposium on Information Visualization (APVIS2006). Tokyo, Japan, 2006. - pp. 207-216.

[4] Isaev R.A., Podvesovskii A.G. Sovershenstvovanie mekhanizma vizualizacii nechetkih kognitivnyh modelej v sisteme podderzhki prinyatiya reshenij «IGLA» [Improvement of the Mechanism of Fuzzy Cognitive Models Visualization in Decision Support System «IGLA»] // Proceedings of the International Conference "Sovremennye tekhnologii v nauke i obrazovanii - STNO2017" [Up-to-date Technologies in Science and Education], Vol.1. - Ryazan: Ryazan State Radioengineering University, 2017. - pp. 138-142 [in Russian].

[5] Meyer B. Self-Organizing Graphs - A Neural Network Perspective of Graph Layout // Whitesides S.H. (eds) Graph Drawing. GD 1998. Lecture Notes in Computer Science, vol 1547. Springer, Berlin, Heidelberg.

[6] Noack A. An energy model for visual graph clustering // Proceedings of the 11th International Symposium on Graph Drawing. Springer-Verlag, 2004. - pp. 425-436.

[7] Podvesovskii A.G., Isaev R.A. Visualization Metaphors for Fuzzy Cognitive Maps // Scientific Visualization, 2018, Vol. 10, Num. 4, pp. 13-29. - doi: 10.26583/sv.10.4.02

[8] Silov V.B. Prinyatie strategicheskih reshenij v nechetkoj obstanovke [Strategical Decision Making in Fuzzy Environment] - Moscow: INPRO-RES, 1995. - 228 p. [in Russian]

[9] Zakharova A.A., Podvesovskii A.G., Isaev R.A. Matematicheskoe i programmnoe obespechenie podderzhki kognitivnogo modelirovanija slabostrukturirovannyh organizacionno-tehnicheskih system [Mathematical and Software Support for Cognitive Modeling of Semi-structured Organizational and Technical Systems] // International conference CPT2019. - Nizhniy Novgorod: Pub. NNGASU and SRCIPT. 2019. - pp. 131141 [in Russian].

[10] Zakharova A.A., Shklyar A.V. Visualization Metaphors // Scientific Visualization, 2013, Vol. 5, Num. 2, pp. 16-24. 